\title{
Cellules contaminées dans Mon frère de Jamaica Kincaid: Le sida circule dans la famille
}

\author{
Bénédicte Boisseron
}

This analysis of Jamaica Kincaid's autobiographical text My Brother relies on the question of a double contamination: AIDS and family. It plays on the relationship between the familiar and the familial, and reflects on the effect of AIDS as an intrusion within the family. Here, AIDS is perceived as the trigger of memory, forcing three traumatic spaces to resurface in the narrator's mind: sexuality, death and family. Bénédicte Boisseron shows how Kincaid's writing closely links AIDS to family and how she manages them both through a mecanism of appropriation and rejection.

Life - blood, sexual fluids - is itself the bearer of contamination. Theses fluids are potentially lethal. Better to abstain.

Susan Sontag, AIDS and Its Metaphor.

Ô petites nations. Dans l'intimité chaleureuse, chacun y envie chacun, tout le monde y surveille tout le monde: " Famille, je vous hais ! » Et encore ces autres mots de Gide: « Rien n'est plus dangereux pour toi que $t a$ famille, que $t a$ chambre, que ton passé [...] Il te faut les quitter.

Milan Kundera, Testaments trahis.

"Le sida ne passera pas par moi ». Le message derrière la fameuse campagne française contre le sida est sans équivoque et associe clairement l'espace ludique et enfantin de la chanson « Furet des bois mesdames ${ }^{1}$ à la contamination du virus. L'analogie entre le monde ludique de l'enfance évocateur de pureté, sécurité, et innocence et celui du sida - univers déchu par excellence - est supposée conduire la conscience collective au cocon protecteur du latex, paradis artificiel de l'innocence retrouvée. Mais, je vous le demande, peut-on vraiment récupérer une innocence perdue? La protection est-elle réellement fiable contre toute fuite de sens? J'ai envie de croire que la protection 
constitue plus qu'un filtre, un isolant, mon isolant; permettant que le monde soit touché mais pas moi, le sida n'est pas passé par moi. Et là, je serais un adulte qui jouerait à l'enfant, celui qui joue à " on fait comme si ", comme s'il était un vendeur de glace ou une maîtresse, un adulte dans un monde d'enfants. Sauf que moi, mon "comme si " serait un univers de cristal qui m'envelopperait d'une couche d'enfance dans un monde trop adulte. Et le cristal serait plus résistant que le fer. " Eh maman, dis voir, qu'est-ce qui est encore plus résistant que le cristal, plus résistant que tout? Tu ne sais pas? » Â ce moment précis, la maman faillit à son devoir de toute puissance des " pourquoi " et des « parce que ». L'enfant prend ainsi brutalement conscience que la famille ne le protègera jamais plus de l'inconnu, du dehors, du dangereux - cette dernière étant elle-même le dehors inconnu. La famille se révèle une imposture qui faisait " comme si " depuis le début.

Mon frère de Jamaica Kincaid, écrivaine américaine originaire d'Antigua, est un récit autobiographique sur la double contamination par le sang: la famille et le sida. Le livre de Kincaid décrit le retour forcé de l'auteur dans son île d'origine, dans sa maison d'enfance, au chevet de son frère mourant du sida. Chaque étape du retour en arrière (île natale/maison familiale/chevet fraternel) fait remonter à la surface une couche différente d'un même traumatisme refoulé. Cet article se propose de démontrer comment ce retour spatial et temporel forcé au point initial s'engouffre dans une traversée aux enfers idéologiques où le sida ramène la narratrice à l'événement primal. Nous allons voir comment la relation sida-frère dans la vie de Jamaica Kincaid fonctionne en tant que « symbole mnémonique » - terme utilisé par Jean Laplanche pour décrire l'événement secondaire qui rappelle l'événement primal réprimé - ramenant à la surface le traumatisme originel que représente la famille. La rencontre familiale précipitée par le sida génère une récurrence, une sorte de rechute de la primo-infection à l'origine $\mathrm{du}$ désaveu fétichiste. Famille/sida, cellules contaminatrices/contaminées, à la vie à la mort. La famille est passée par moi.

Nous apprenons que la narratrice de Mon frère n'était pas retournée à Antigua et n'avait pas parlé à sa famille depuis des années avant ce jour déclencheur du récit. Kincaid nous dit qu'elle avait pris l'habitude de se distancier régulièrement de sa famille afin de s'oxygéner et d'éviter que la proximité trop régulière avec les membres de sa famille la rende beaucoup trop vulnérable à leur demande et influence imposantes. Ce jour fatidique, elle reçoit un coup de téléphone qui lui apprend que son petit frère est en train de mourir du 
sida. À partir de ce moment, écrit-elle:

[...] la réflexion que je m'autorise d'ordinaire chaque fois que se présentent les besoins de ma famille - convient-il ou non que je laisse cela $\mathrm{m}^{\prime}$ affecter? - se volatilisa. J'ai eu l'impression de tomber dans un grand trou et je n'ai pas cherché à arrêter ma chute. J'ai eu l'impression d'être engloutie dans une grande vapeur de tristesse et je n'ai pas cherché à y échapper. (24)

Suivant le rapprochement forcé qui s'opère après la nouvelle de la maladie, la structure de Mon frère se construit sur un face à face insolite entre le sida et la famille. La narratrice, en proie à une tension fusionnelle d'une violence à peine retenue, se voit coincée entre la cellule infectée par le virus et la cellule familiale contaminatrice. La famille et le sida, deux impostures dans la vie de la narratrice, sont mis à nu et imposent une trêve à ce jeu faussement protecteur de « comme si ».

Kincaid nous fait entrer dans un monde post-traumatique, éminemment cru et dépourvu de désaveu. Octave Mannoni, dans Clefs pour l'imaginaire, utilise la formule bien connue "Je sais bien mais quand même... " pour décrire le désaveu. Ce fétichisme primaire consiste à réfuter la conscience (bien acquise et formulée par « Je sais bien ») de la réalité par l'intermédiaire d'une attitude d'autruche (exprimée par " mais quand même ») qui permet d'agir " comme si » on ne savait pas. Mannoni prend appui sur les sociétés primitives ainsi que sur l'enfance afin de démontrer le mécanisme du désaveu dans sa forme la plus pure, primaire, et totémique. Le père Noël, par exemple, figure magique de l'enfance, est très tôt décelé par l'enfant comme une imposture, un faux-semblant frauduleux manigancé par les adultes. L'enfant, cependant, s'accroche à son innocence infidèle. Il commence à agir "comme si » l'oncle caché derrière la barbe blanche bon marché qu'il a vue toute l'année rangée dans le placard était bien le père Noël. Continuerait-il à recevoir des cadeaux s'il n'y croyait plus? Plus tard, il fera " comme si » ce bout de papier, sur lequel est écrit « 20 euros " et qui s'appelle "billet de banque ", a une valeur telle qu'il peut être échangé contre de la marchandise. Comment se procurerait-il cette marchandise s'il cessait d'y croire? Le monde adulte n'est qu'une continuité de fétichismes primaires et enfantins grâce auxquels l'être peut faire « comme si » le monde était un lieu magique. Les premiers traumatismes se nichent dans la plus tendre enfance, là où la famille s'efforce de faire croire à un monde fabuleux où la mort, la 
sexualité et la peine n'existent pas. Le mécanisme de protection chez l'enfant l'amène à gérer la conscience progressive du leurre familial, et par extension du monde adulte, avec un désaveu fétichiste de « comme si ». Chaque futur désaveu ne sera qu'un rejeton du traumatisme originel lié à la conscience de la famille comme imposture. $\mathrm{La}$ famille, un traumatisme? Je sais bien mais quand même...

Dans le livre de Jamaica Kincaid, le lecteur découvre comment le désaveu fétichiste s'effectue au quotidien dans son rapport à la famille. Il s'inscrit comme primo-infection qui crée des anti-corps pour le système immunitaire de négation. Ce système pourra être appliqué par la suite dans tous les autres événements source de traumatisme. Le tabou de la sexualité au sein de la cellule familiale est central dans la formation des manifestations fétichistes de l'adulte. Les parents font " comme si » ils n'avaient pas d'organes génitaux et de rapports sexuels afin de préserver leurs enfants dans cette boule de cristal au futur goût de latex. Le tabou du sexe nous amène à croire que la famille n'a pas droit à la sexualité. Elle est le cocon de protection qui s'est conçu par la copulation mais qui rejette son appropriation à l'intérieur de sa cellule. $Q u^{\prime}$ arrive-t-il alors quand Devon, le frère de Kincaid, meurt du sida, maladie sexuellement transmissible? Et comment réagir quand ce frère vient vous jeter à la figure, sans vous demander votre avis, la vue de son sexe purulent contaminé par la maladie? Le traumatisme originel de la famille devient tout à coup indissociable du sida, de la vie et de la mort, de l'abstinence et de la sexualité. Devon a brisé la couche de protection enfantine du "comme si » en imposant à sa sœur la vision d'un pénis stigmatisé par la maladie et la mort, «sans m'avoir préparée à voir son pénis» dit-elle, " je ne voulais pas voir son pénis» (87).

" Jamaica, regarde-moi ça, mais regarde-moi ça » (86). La signification de cet affichage du pénis fraternel marqué par la maladie - acte de désespoir et de provocation - siège au-delà de l'idée dérangeante de la mort dans le sida. Il rend compte du rapprochement forcé de deux entités apparemment non familières mais secrètement hantées par le malaise du familier: la sexualité et la mort. Tel le Unheimlich freudien, ce quelque chose secrètement familier qui fait son entrée sous une allure de non familier, ces deux entités normalement familières, une fois réunies par le même signifiant « sida », s'annoncent insolites. L'effet insolite n'est autre que cette résurgence de deux occurrences vécues dans l'enfance, au sein de la famille, "comme si » elles n'existaient pas. Le désaveu imposé par la famille, ce « comme si » protecteur, se donne des airs de familier, d'ordinaire et d'entendu. Seul 
l'événement secondaire aux relents anamnestiques rappelle son caractère non familier et traumatique réprimé ${ }^{2}$. Une fois que le symbole mnémonique prend place - la mort du frère du sida - tout ce qui avait pu être réprimé sous la prétendue familiarité s'affiche soudainement comme non familier et insolite. "Tout ce qui était étranger se faisait dedans, tout ce qui était familier et important se faisait dehors " (108) écrit Kincaid. Et c'est justement le support familial/familier chez l'auteur qui expose l'insolite de la sexualité accouplée avec la mort dans le sida. Le noyau famille/mort/sexualité est révélé dans tout son caractère de traumatisme primal.

La mort, tout d'abord, est perçue par la narratrice avec un œil entraîné maintenant à réfuter toute protection de " comme si »:

Ce genre de comportement, ce genre de sentiment, si hystériques, si tristes, quand quelqu'un est mort, ne sont pas du tout de mon goût et je voudrais les éviter. Ce n'était pas comme si [mes italiques] tout ça n'était encore jamais arrivé, ce n'est pas comme si [mes italiques] les gens ne mouraient pas depuis toujours [...] Pourquoi tout un chacun ne peut-il s'y habituer? (130)

Ce conditionnement à renier la mort est une séquelle de la protection familiale solidement incrustée dans les consciences, un reste de l'enfance de " elle ne passera pas par moi ». Kincaid prend conscience que non seulement les gens agissent « comme si » la mort n'existait pas, " comme si » ils ne l'avaient jamais rencontrée sur leur passage, mais aussi qu'elle-même, la narratrice, ne peut échapper à cet effet inéluctablement amnésique de l'expérience de la mort. La mort prend à contre-courant et sa fonction javellisante, cette façon qu'elle a d'éviter toute trace $d$ 'infection derrière elle, lui permet de toujours intervenir contre toute attente:

La mort est ainsi. Elle se produit tous les jours mais quand on voit des gens en deuil, ils se comportent comme si [mes italiques] c'était si nouveau, cet événement mourir - quelqu'un qu'on aime meurt - cela n'était encore jamais arrivé; c'était si inattendu, si injuste, unique pour nous. (116)

Le sida est une maladie qui contamine non seulement les cellules saines du corps organique mais qui s'attaque aussi au corps signifiant de la représentation symbolique. Dans Facing It, Ross Chambers parle d'une écriture de "thinning out » - de rétrécissement jusqu'à 
extinction - de la victime du sida qui témoigne de son dépérissement, son amaigrissement, son lent et insolent voyage au bout de la mort. Le sida, en tant que maladie du rétrécissement, s'accompagne d'une diminution du signifiant « sida ». Maladie mortelle dévorant le corps, le sida est de moins en moins maladie et de plus en plus mort. Le malade du sida, dans son revêtement progressif de la mort, devient cadavre vivant. Il est chute (cadere, tomber), comme le souligne Julia Kristeva, ne renvoyant pas à la mort référant (comme le serait un encéphalogramme précise Kristeva), mais la mort dans toute sa matérialité de référé: "Une plaie de sang et de pus, ou l'odeur doucereuse et âcre d'une sueur, d'une putréfaction, ne signifient pas la mort. [...] Non, tel un théâtre vrai, sans fard et sans masque, le déchet comme le cadavre $\mathrm{m}^{\prime}$ indiquent ce que j'écarte en permanence pour vivre » (11).

Et en permanence me voici écartant la mort, la famille, et la sexualité contaminée pour vivre, car elles ne passeront pas par moi.

La sexualité, liée à la mort dans son rapport au sida, se voit tout aussi insolite dans sa relation au familier. Kincaid écrit:

Les gens là d'où je viens sont tout à fait à l'aise avec la honte de la sexualité, de son inexplicable besoin, d'une façon d'en jouir qui semble excéder l'ordinaire [mes italiques], de sa réelle particularité; c'est seulement quand on en meurt, de la sexualité, que la honte devient, disons, une honte. (174)

Ce n'est pas la maladie en tant que telle qui ôte à la sexualité son voile et sa protection de normalité. C'est le sida, cette maladie rassemblant dans un signifiant unique la sexualité et la mort, qui produit l'impossibilité du désaveu fétichiste. L'occurrence de cette maladie mortelle à forte connotation sexuelle chasse la mort et la sexualité hors scène et les rend du coup "ob-scènes ", " excédant l'ordinaire " comme nous dit Kincaid. L'espace idéologique ne remplit plus son devoir de désaveu fétichiste et refuse dès lors de couvrir la sexualité et la mort d'une image rassurante de familiarité. Du même coup, la cellule familiale, ce foyer accueillant et recueillant le sida, perd toute protection idéologique et dévoile son visage obscène, rappel du traumatisme originel.

Le récit de Jamaica Kincaid est composé principalement de haine/amour pour sa famille, particulièrement pour cette mère qui représente la plus grande imposture de sa vie. Chaque petit détail que 
la narratrice consacre aux souvenirs d'enfance remplis de rancoeur et de douleur (le jour où sa mère a fait un feu de joie avec ses livres, par exemple, ou quand elle l'a retirée de l'école pour qu'elle s'occupe des tâches domestiques à la maison) n'est qu'une particule d'une cellule contaminée bien plus générique: la famille. La famille, comme la maladie du sida, vous tient par les liens du sang et vous consume lentement de l'intérieur. Elle expose les stigmates de cette consommation sur le tas, à l'heure où le système immunitaire de désaveu est trop affaibli pour continuer à vous protéger de son " comme si ».

À partir du moment où Kincaid reçoit ce coup de téléphone d'Antigua lui annonçant la maladie de son frère, cette femme qui se voulait indépendante, protégée de sa famille par la distance géographique et par son rôle de mère et de femme mariée, subit une rechute dans la sphère familiale primale. La voici, à plusieurs reprises, de retour à Antigua, au chevet son frère moribond - ce frère encore dans la maison familiale, aux crochets d'une mère vampirique prenant soin de ce corps à la vulnérabilité enfantine en pleine décroissance. Il y a, dans ce retour imposé à Antigua, un lien symbolique étroit entre le pays et la famille. La narratrice réserve à ces deux formes de retour au bercail le même traitement, jouant sur l'insolite et réveillant chez le lecteur un système de défense qui l'oblige à reconsidérer ses propres repères idéologiques ancrés dans le désaveu.

La famille, protégée par la répression du traumatisme originel grâce au désaveu fétichiste, se voit attribuée une image de bien-être et de " chez soi » par le schème idéologique. Cette matrice réconfortante est magnétique dans son appel au retour fœtal. Or, quand cet appel prend des tournures de mante religieuse mauriacienne sous la plume de Kincaid, c'est l'édifice idéologique entier, douanier de la frontière familier/insolite et seul détenteur de la répression des origines, qui s'écroule. La famille, aussi bien que le pays natal, sont soudainement vus sous la lumière crue de l'absence de désaveu. Est restitué à Antigua son étrangeté, ce malaise qui suit le retour au pays d'origine après une absence prolongée; et la famille retrouve ce sens insolite, cette gêne qui s'attache à la cellule une fois le cordon ombilical coupé. Quand la famille et la patrie sont exposées dans toute leur nudité dépourvue d'illusion idéologique, l'être "trébuche sur lui-même " comme dirait Philippe Sollers (183). Le trébuchement identitaire sur soi arrive quand on commence à regarder où l'on marche et où l'on est. C'est l'étourdissement des origines.

Inspirée par le roman sur l'immigration intitulé Ignorance du franco-tchèque Milan Kundera, je suis tentée de nommer "syndrome 
Calypso » le malaise que Kincaid décrit dans Mon frère. Kundera s'interroge sur cette nostalgie, ce manque profond du pays, du foyer, qui a forcé Ulysse à quitter les bras de Calypso après sept longues et heureuses années d'exil, pour retrouver ceux de Pénélope au pays d'origine. Au lieu de l'inconnu et de l'étranger, s'étonne Kundera, Ulysse a préféré le confort du connu, du familier, de la maison. Une fois Ulysse rentré au bercail, cependant, les siens ne le reconnurent même pas, le flair de son chien étant seul capable de sentir le familier en famille. Ce phénomène aliénant de la perte du familier dans la famille/patrie se nomme, en allemand, le Die Entfremdung. Dans Testaments Trahis, Kundera explique ce processus « durant lequel ce qui nous a été proche est devenu étranger » :

On ne subit pas l'Entfremdung à l'égard du pays d'émigration: là le processus est inverse: ce qui était étranger devient, peu à peu, familier et cher. L'étrangeté dans sa forme choquante, stupéfiante, ne se révèle pas sur une femme inconnue qu'on drague, mais sur une femme qui, autrefois, a été la nôtre. Seul le retour au pays natal après une longue absence peut dévoiler l'étrangeté substantielle du monde et de l'existence. (117)

La distance géographique permet de préserver un " comme si » protégeant l'image familière de la famille. Une fois cette distance comblée par le retour au nid, le sevré se retrouve dans l'impossible entre-deux d'une proximité physique qui ne fait que rendre plus évidente la distance idéologique aliénante qui le sépare du lieu natal. On découvre, dans L'Odyssée d'Homère, l'origine même du rapport de l'insolite en famille comme on peut l'observer aussi dans le livre de Kincaid. Une fois parti, le retour au nid n'est plus possible car le nid aussi bien que le fils prodigue ont perdu tout sens de familiarité. Kincaid observe son pays et sa famille avec les yeux d'un imposteur qui voit la famille comme étant elle-même une imposture. La famille se situe alors dans un hors-champ idéologique où seul le lien ombilical, ce sang qui coule d'une cellule à l'autre, la forcera à expier sa peine d'infidélité familiale et d'identité nationale par l'écriture. Mon frère est un récit autobiographique par procuration ${ }^{3}$ qui reprend à son compte la maladie et la mort de son frère. Cette prise en compte s'opère non par une écriture témoin au ton élégiaque mais par une écriture d'accusée, une écriture qui reconnaît sa contamination du sida et de la famille tout en avouant ne pas avoir voulu qu'elle passe par elle. "J'ai commencé à ressentir que ce serait si bien qu'il se décide à mourir tout de suite, dit Kincaid, 
pour être enterré tout de suite et puis voilà » (86). Se débarrasser au plus vite de la contamination famille/sida/patrie, comme si le virus n'existait plus une fois les symptômes disparus.

Dans le roman de Kundera, nous pouvons retrouver le même type de personnage dans Irène, cette femme tchèque qui, après dix-sept ans d'exil en France, sans famille, rejoint sa mère en Italie. " Mais n'étaitce pas horrible de la part de la fille " écrit Kundera en style indirect libre, « de n'être pas heureuse de la présence de sa mère qui, après dix-sept ans d'exil venait lui rendre visite " ? Cette question devient encore plus intéressante quand elle est apposée à celle du retour au pays natal. Après la chute du communisme en Europe de l'Est en 1989, les exilés politiques se voyaient soudainement changer de statut dans l'opinion publique. Ils étaient avant lors considérés avec des yeux mouillés de compassion et de sympathie étant orphelins sans patrie. Mais le mur idéologique est tombé, l'obstacle du retour au pays a disparu du même coup. Et là, les yeux accusateurs ont commencé à se poser des deux côtés de cette barrière maintenant fantomatique. Pourquoi ne pas rentrer voir leur pays et leur famille? Oui, en effet, Irène maintenant se demande aussi pourquoi. Kundera explique ainsi qu'Irène avait toujours vu l'émigration associée à la notion de malchance. Elle avait donc toujours été convaincue que l'association émigration/malheur coulait de source. Mais maintenant, dans cette nouvelle donnée idéologique et historique qui la contraignait à rentrer " chez elle ", elle commençait à se demander si, en fait, cette vision négative de son émigration n'était pas le fruit d'un conditionnement extérieur imposé par ces indigènes qui vous regardent avec des yeux de pitié, vous laissant entendre que votre exil est à plaindre. Non, avait-elle décidé, son exil était une chance, le bonheur de la liberté offerte contrainte et forcée. Est-ce l'image du pays d'origine imposée par la pression idéologique qui nous fait croire que les bras de Pénélope sont préférables à ceux de Calypso? Devrait-on se sentir coupable d'infidélité dans sa recherche du plaisir le plus loin possible, dans cette quête de la ville étrangère où il est nécessaire d'abandonner ce besoin de familiarité et de tenir les choses à distance, de les traiter en étranger afin de mieux les posséder? Famille, patrie: êtes-vous Pénélope ou Calypso?

L'intrusion de la famille dans la vie d'expatriée de Kincaid - qui dédouble l'intrusion virulente de la maladie dans le petit confort de sa vie dans le Vermont - $s$ 'impose jusque dans les fibres génétiques de 
son discours. L'utilisation, d'une fréquence inhabituelle et envahissante de l'expression " mon frère " pour désigner tous ses frères indique une intention, de la part de la sœur, de ne voir autre chose dans sa famille qu'un lien congénital. La présence de « mon frère " tourne à l'obsessionnel et stigmatise l'écriture de Kincaid au point de nuire à la fluidité de lecture. Ce refus presque imperturbable $\mathrm{d}^{\prime} u$ tiliser les noms propres pour désigner ses frères produit un langage symptomatique de traumatisme. Kincaid ne peut surmonter la pression du lien familial et cet échec transparaît dans son écri-ture qui se trouve contaminée par la présence familiale. Le lecteur est ainsi submergé par des phrases aux propositions relatives consécutives indigestes: « $[\ldots]$ mon frère qui était mort, mon frère qui était marchand au marché le samedi, mon frère qui avait presque tué notre mère en la jetant par terre pour essayer de lui lancer des pierres " (98). Seulement une seule fois Kincaid mentionnera le prénom de ses deux autres frères lors du récit. Son écriture est significative d'une volonté de réduire sa famille à une fonction symbolique. Un tel recours rhétorique lui donne accès à un pouvoir de distanciation produit par cet art de grillager son espace vital de statuts purement génériques. N'étant attribuée d'autre fonction que celle qu'elle représente, la famille se voit déshumanisée et défamiliarisée ${ }^{4}$. La famille est passée par Kincaid mais cette dernière ne leur aura cédé, mesquinement, que ce qu'il leur revenait par droit du sang, c'est-à-dire le rapport congénital. On peut rejeter sa mère ou sa famille mais l'acte même de rejet reconnaît et s'approprie, dans une logique perverse, l'affiliation. En rejetant mon frère, je le fais mien par les droits du sang. Mon rejet de la famille et de la maladie qui l'a contaminée me lie à elles plus étroitement. Famille/sida/mort m'attendent à Samarkande...

Jean Baudrillard, dans De la séduction, nous raconte l'histoire de ce soldat qui rencontre la mort lui faisant signe au coin d'une rue à la sortie du marché. Pris de panique, ce dernier court au palais demander au roi son meilleur cheval afin de s'enfuir au plus vite. Il est parti, loin, très loin, jusqu'à ce havre de sécurité, Samarkande. Le roi convoque la Mort pour lui reprocher d'avoir fait peur à son soldat par ce signe de la main et la Mort lui répond: « Je n'ai pas voulu lui faire peur. C'était seulement un geste de surprise, de voir ici ce soldat, alors que nous avions rendez-vous à Samarkande » (101).

Selon Susan Sontag, l'être moderne a perdu la force d'aller fuir et rejoindre la mort à Samarkande. Il vit aujourd'hui, ou plutôt survit, dans un état constant de négation, refusant de voir l'épée de Damoclès. Cependant l'être a conscience que cette épée peut l'atteindre à tout 
moment. Susan Sontag, dans " AIDS and Its Representation », voit cette négation du glaive symptomatique des temps modernes et de son entre-deux événementiel. Le monde actuel a vu sa structure organique se déplacer lentement vers une logique, selon Sontag, $d^{\prime}$ " Apocalypse From Now On » (176). Chaque événement en soi dramatique n'est jamais l'événement apocalyptique dans toute son ampleur mais seulement son déclenchement, ou son avertissement. En d'autres termes, la réalité de l'événement, comparée à ce qu'elle sera ou ce qu'elle pourra être - d'après les statistiques et pronostiques scientifiques - n'est vraiment perçue que dans sa croissance exponentielle. «There is what is happening now. And there is what it portends: the imminent, but not yet actual, and not really graspable, disaster » (178). Comme l'explique Sontag, la différence entre l'épidémie et la pandémie prévue (les chiffres à venir de l'invasion à l'échelle globale de la maladie), rend compte de la même différence entre les guerres que nous avons (contenues et pas encore nucléaires) et les guerres bien plus destructives que l'on pourrait avoir. C'est dans ce décalage entre l'événement réel et sa projection virtuelle et scientifique à tonalité apocalyptique que vient se nicher une certaine forme de désaveu fétichiste. L'appréhension de la réalité dramatique dans sa projection apocalyptique - ce que Sontag nomme le " future mindedness " (177) - permet à l'être de continuer de vivre normalement dans un esprit de " en attendant ", " comme si » il était encore protégé pour l'instant.

Dans cette parenthèse où l'idée de l'apocalypse repoussée à plus tard peut faire partie de la violence ordinaire du quotidien, l'être arrive à désavouer sa réalité. C'est dans cette projection de l'apocalypse que la réalité elle-même apocalyptique passe inaperçue. La réalité traumatisante peut être survécue uniquement dans son déplacement, vers son ailleurs, son plus tard. Selon Sontag, le sida n'échappe pas à cet entredeux événementiel qui appartient à un futur " which is already here and always before us, which no one knows how to refuse " (181). L'auteur précise qu'il serait désirable que, dans l'avenir, quand on pourra mieux le gérer et le comprendre, le sida vienne rejoindre le monde ordinaire d'une maladie comme une autre.

Quand/si le sida venait à devenir une maladie ordinaire, ne seraitil pas géré comme la famille, la mort et la sexualité: enfoui dans un désaveu fétichiste? La maladie insolite par excellence s'offrirait alors des allures familières afin de mieux cacher son ailleurs, son inconnu, son traumatisme originel sous-jacent lié à sa connotation de mort sexuelle et de sexualité mortelle. Traité le sida comme on traite le traumatisme de la famille reviendrait à ce que chaque membre de la cellule 
contaminée agisse « comme si » il était une maladie ordinaire. Le sida ne jouerait plus le rôle de support du Unheimlich freudien, exposant l'insolite de la famille, de la mort, et de la sexualité, tous trois rassemblés dans la même cellule contaminée sur le lit de mort de Devon, le frère de Jamaica Kincaid. Et après tout, pourquoi pas? Pourquoi ne pas faire comme si le sida faisait partie de la cellule familière puisque, Samarkande ou pas, le sida est passé par nous?

\section{Notes}

${ }^{1}$ La campagne française a repris les paroles de la chanson "Le furet des bois mesdames" en remplaçant le furet par le sida donnant: "Il court, il court le sida. Il est passé par ici, il repassera par là". Voici les paroles d'origine:

Il court, il court le furet

Le furet des bois Mesdames

Il court, il court le furet

Le furet des bois jolis

Il est passé par ici

Il repassera par là

* Merci aux deux générations de mères Boisseron (Sophie et maman) de $m^{\prime}$ avoir rappelé avec exactitude les paroles de cette chanson enfouies dans ma mémoire d'enfant.

2 Dans son article "The Uncanny ", Freud explique que " the uncanny is in reality nothing new and alien, but something which is familiar and old-established in the mind and which has been alienated from it only through the process of repression » (241).

3 Voir Facing it de Ross Chambers (Ann Arbor: The University of Michigan Press, 1998) pour un glossaire de certains termes d'écriture sur le sida.

4 Dans "The Uncanny ", Sigmund Freud explique que dans une situation insolite « a symbol takes over the full function of the thing it symbolizes " (242); c'est exactement ce qu'on peut observer dans l'écriture de Kincaid. L'écrivain rend volontairement sa famille insolite et privée de familiarité en hypertrophiant la fonction symbolique aux dépends du référé. 


\section{Bibliographie}

Baudrillard, Jean. De la séduction. Paris: Galilée, 1979.

Chambers, Ross, Facing It. Ann Arbor: The University of Michigan Press, 1998.

Freud, Sigmund. "The Uncanny ». The Standard Edition of the Complete Psychological Works of Sigmund Freud Vol XXII. London: Hogarth Press, 1953.

Kincaid, Jamaica. Mon frère. Traduit par Jean-Pierre Carasso \& Jacqueline Huet. Mon frère. Paris: Editions de l'Olivier/Seuil, 2000. Kristeva, Julia. Pouvoirs de l'horreur. Paris: Seuil, 1980.

Kundera, Milan. Ignorance. NY: Harper Collins Publishers, 2002.

- Testaments Trahis. Paris: Gallimard, 1993.

Laplanche, Jean. "Sexuality and the Vital Order » Life and Death in Psychoanalysis. Baltimore / London: John Hopkins University Press, 1985.

Mannoni, Octave. "Je sais bien mais quand même... " Clefs pour l'imaginaire. Paris: Seuil, 1968.

Sollers, Philippe. Femmes. Paris: Gallimard, 1983.

Sontag, Susan. Illness as Metaphor and AIDS and Its Metaphor. New York: Picador, 1977. 